\title{
Para una Bibliografía de y sobre Severo Sarduy (1955-1971)
}

Los medios de difusión son hoy tan abrumadoramente amplios que sería insensato no resignarse a la imposibilidad de compilar la bibliografía completa de un escritor contemporáneo. $L a$ que of rezco a continuación, sin embargo, es lo más completa posible dentro de esos límites. Además de las fuentes bibliográficas usuales, tuve acceso en París a los archivos personales de Severo Sarduy (a quien agradezco el favor) y a los del servicio de prensa de Editions du Seuil, donde trabaja el escritor y donde se han publicado las traducciones francesas de sus novelas. En la biblioteca de la Universidad de Yale (de la mejor es de los Estados Unidos en literatura cubana reciente) pude consultar Lunes de Revolución y otras publicaciones de la época (1959-60) en que colaboró Sarduy; de este período la única laguna es el Diario Libre, en el que Sarduy dirigió una página literaria por algún tiempo y que me ha sido imposible localizar. También en Yale consulté Ciclón, pero sólo pude completar esa sección con la ayuda del trabajo de Gary Brower y Raymond D. Souza, "Ciclón, índice e introducción", Et Caetera, Año 4, 1959, pp. 105-25.

He preferido organizar cronológicamente la bibliografía sobre Sarduy para dar idea del desarrollo de la crítica sobre su obra; siendo ésta rela. tivamente modesta todavia, dada la juventud del escritor, no creo que esta desviación del formato usual sea un obstáculo infranqueable para el lector habituado al orden alfabético. Con el propósito de jalonar la producción de Sarduy he puesto bajo rúbrica aparte lo publicado en varias revistas importantes: Tel Quel, Mindo Nuevo, Lunes de Revolución, etc. Por último, en dos o tres ocasiones me ha sido imposible obtener el número de la página en que aparece un artículo que sólo he podido ver en forma de recorie de periódico.

Comell University

Roberto GonzÁlez ECHEVARRÍA 


\section{BIBLIOGRAFIA SOBRE SEVERO SARDUY}

\section{LIBROS}

1. Gestos. Barcelona: Scix Barral, 1963. [Novela.]

2. De donde son los cantantes, México: Joaquín Mortiz, 1967. INovela.]

3. Escrito sobre un cuerpo. Ensajos de crítica. Buenos Aires: Sudamericana, 1969.

4. Flamenco. Grabados de Erhardt. Stuttgart: Manus.Presse, 1969. [Libro-objeto, poemas.]

5. Mood Indigo. Grabados de Erhardt. Stuttgart: Manus-Presse, 1970. [Libro-objeto, poemas.]

6. Merveilles de la Nature. Ilustraciones de Leonor Fini. París: J. J. Pauvert, 1971. [Libro-objeto, poemas.]

\section{TRADUCCIONES DE LAS NOVELAS}

De GESTOS:

7. Gestes. Tr. Henri Sylvestre. París: Editions du Seuil, 1963.

8. La bomba dell'Avana. Tr, Alvar González Palacios. Milán: Feltrinelli, 1964.

9. Kapricer. Tr. Ebbe Traberg. Copenhague: Grafisk Forlag, 1964.

10. Gesty. Tr. Mikolaj Bieszczadowski. Varsovia: Instytut Wydawniczy, 1965.

11. Beuegungen. Erzäblung. Tr. Helmut Frielinghaus. Frankfort: Suhrkamp, 1968.

De DE DONDE SON LOS. CANTANTES:

12. Ecrit en dansant. Tr. E. Cabillon, Cl. Esteban y el autor. París: Editions du Seuil, 1967.

\section{COLABORACIONES EN LIBROS, PERIODICOS Y REVISTAS}

En CiclóN (La Habana) :

13. "Poema", 1 [No. 4] (1955), p. 40. 
14. "Sobre el infierno", 2 [No, 1] (1956), pp. 54.56." [Reseña de Jean Guitton, El infiemo.]

15. "Poemas", 2 [No. 3] (1956), p’p. 49.50. ["Islas", "La ventana" y "Angeles."]

16. "Fábulas", 2 [No. 6] (1956), pp. 52-53. [Poemas.]

En NUEVA GENERACION, página literarid del periódico REVOLUCION (La Habana):

17. "Dos décimas revolucionarias", 13 de enero de 1959, p. 5.

18. "Las bombas", 19 de enero de 1959, p. 15. [Ensayo-relato.]

19. "El general", 27 de enero de 1959, p. 15. [Relato.]

20. "En su centro", 28 de enero de 1959, p. 15. [Ensayo sobre José Martí en número especial conmemorando su natalicio.]

21. "Pintura y revolución", 31 de enero de 1959, p. 14.

22. "El torturador", 6 de febrero de 1959, p. 15. ['Cuento cubano'.]

23. "Contra los críticos", 16 de febrero de 1959, p. 16.

En LUNES DE REVOLUCION, Suplemento literario del periódico REVOLucion (La Habana):

24. "De este modo: homenaje a Ballagas", No. 26, 14 de septiembre de 1959, p. 11. [Dos sonetos en número especial dedicado al au. tor de Sabor eterno en el que también colaboran José Lezama Lima, Virgilio Piñera, Roberto Fernández Retamar, Heberto Padilla y otros.]

25. "La revolución de un pintor: homenaje a Víctor Manuel", No. 29, 5 de octubre de 1959, pp. 8-9.

26. "En casa de Mariano", No. 30, 12 de octubre de 1959, pp. 3-5.

27. "En el Salón Nacional de pintura y escultura", No. 31, 19 de octubre de 1959 , pp. 2.4.

28. "La taza de café", No. 38, 7 de diciembre de 1959, p. 16. [Crítica de teatro.]

29. "La bienal de Venecia", No. 65, 27 de junio de 1960, p. 23. [Reportaje desde Europa.]

30. "Picasso expone", No. 72, 15 de agosto de 1960, pp. 16.17.

31. "'Amor es decir ven a mi casa' y 'Nacer es entrar en una luz violenta" ", Número especial dedicado a los escritores de Camagüey. [Sin número.] 19 de septiembre de 1960, p. 11. [Poemas.] 
En TEL QUEL (Paris):

32. "Pages dans le blanc", No. 23 (1965), pp. 83-88. [Poemas.]

33. "Sur Gongora", No. 25 (1966), pp. 91-93. [Ensayo.]

34. "Cubes", No. 32 (1968), pp. 86-87.

35. "Cobra", No. 43 (1970), pp. 37:46. [Fragmento de la novela inédita del mismo nombre, traducido al francés por Philippe Sollers en colaboración con el autor.]

36. "Tanger", 47 (1971), pp. 86-88. [Texto crítico-poema, traducido por Philippe Sollers y el autor.]

En MUNdo Nuevo (Paris):

37. "De la pintura de objetos a los objetos que pintan", No. 1 (Julio 1966), pp. 60-62. Escrito sobre un cuerpo, pp. 102-08.

38. "Junto al río de las cenizas de rosa", No. 5 (Noviembre 1966), pp. 4-9. De donde son los cantantes, pp. 25-33.

39. "Ernesto Sábato: por una novela metafísica", No. 5 (Noviembre 1966), pp. 5-21. [Entrevista de Sábato con Emir Rodríguez Mo. negal en la que participa Sarduy.]

40. "Sobre Góngora: la metáfora al cuadrado", No. 6 (Diciembre 1966), pp. 84-86. Escrito sobre un cuerpo, pp. 55-60.

41. "Nuestro Rubén Darío", No. 7 (Enero 1967), pp. 33.46. [Diálogo en el que participan Sarduy, Rodríguez Monegal y Tomás Segovia.]

42. "Textos libres y textos planos", No. 8 (Febrero 1967), p. 38. Escrito sobre un cuerpo, pp. 99-101.

43. "Del Yin al Yang (sobre Sade, Bataille, Marmori, Cortázat y Elizondo)", No. 13 (Julio 1967), pp. 4-13. Escrita sobre un cuerpo, pp. 9.30 .

44. "Rosado y perfectamente cilíndrico", No. 16 (Octubre 1967), pp. 24-27. De donde son los cantantes, pp. 36-44.

45. "Un fetiche de Cachemira", No. 18 (Diciembre 1967), pp. 87-91. Escrito sobre un cuerpo, pp. 31-41.

46. "Escritura/travestismo", No. 20 (Febrero 1968), pp. 72.74. Escrito sobre un cuerpo, pp. $43-48$.

47. "Dispersión/falsas notas (homenaje a Lezama)", No. 24 (Junio 1968), pp. 5-17. Escrito sobre un cuespo, pp. 61-89.

48. "Por un arte urbano", No. 25 (Julio 1968), pp. 81.83. Escrito sobre un cuerpo, pp. 93-96. 
En SUR (Buenos Aires):

49. "Poemas bizantinos", No. 291 (Noviembre.diciembre 1964), pp. 55.59.

50. "Curriculum cubense", No. 297 (Noviembre-diciembre 1965), pp. 42-49. De donde son los cantantes, pp. 11-21.

51. "Teatro lírico de muñecas", Nos. $316-317$ (Enero-abril 1969), pp. 24-37. [Fragmento de Cobra.]

52. "Boquitas pintadas: parodia e injerto", No. 321 (Noviembre-diciembre 1969), pp. 71-77.

En LA QUINZAINE LITTÉRAIRE (Paris):

53. "Les dieux et les choses", No. 46 (1968), pp. 18.19. [Reseña de Miguel Barnet, Esclave à Cuba; Bícgrapbie d'un cimarron du colonialisme à l'indepéndance.]

54. "Góngora le baroque", No. 49 (1968), pp. 12-13. [Reseña de Robert Jammes, Etudes sur l'oeuve poétique de Don Luis de Góngora y Argote.]

55. "Anamorphoses", No. 50 (1968), p. 5. [Reseña de Julio Cor. tázar, Gîtes.]

56. "Le grand moment de l'architecture espagnole", No. 53 (1968), pp. 16-17. [Reseña de Damien Bayon, L'Arcbitecture en Castille au $X V I$ siècle.]

57. "L'Objet-fétiche", No. 55 (1968), p. 4 [Reseña de Carlos Fuen. tes, Zone sacrée y Chant des aveugles.]

58. "L'Ecriture autonome", No. 63 (1968), pp. 3-4. [Reseña de Gabriel García Márquez, Cent ans de solitude. Traducción inglesa en Review 70, ed Ronald Christ, Nueva York: Center for InterAmerican Relations, 1971, pp. 171-74.]

59. "Un Proust cubain", No. 115 (1971), pP. 3.4. [Reseña de José Lezama Lima, Paradiso.]

60. "Entre les 'arrivés' et l'avant garde", No. 126 (1971), pp. 13-14.

En publicaciones diversas:

61. "Fábulas", Colección de poetas de la ciudad de Camagïey, ed. Samuel Feijoo. La Habana: Ediciones del Grupo Yarabey, 1958, pp. 54-59. 
62. "Cautelas", Nueva Revista Cubana (Santa Clara), Año 1 [No. 1] (1959), pp. 110-11. [Poemas.]

63. "La Quadriennale de Roma", Artes Plasticas (La Habana), 2 de enero de 1961. [Páginas sin numerar.]

64. "Poesie bizantine", Tempo Presente (Roma), Año 8 [Nos. 3.4] (1963), pp. 65-66.

65. "Peintres et machines", France Observateur, No. 754 (1964).

66. "Azahara", Zona Franca (Caracas), Año 1 [Nos. 13.14] (1965), pp. 22.23. [Poemas.]

67. "En el bosque de La Habana", Zona Franca (Caracas), Año 1 1965). De donde son los cantantes, pp. 25-27.

68. "Con fondo verde y gritando", Diálogos (México), 1 [No. 6] (1965), pp. 15-17. De donde son los cantantes, pp. 117-25.

69. "Del ying al yang", Zona Franca (Caracas), Año 2 [No. 30] (1966), pp. 14-15. [Poemas.]

70. "Las poco-pelo", Papeles de Son Ammandans, No. 123 (1966), pp. 303-20. De donde son los caniantes, pp. 103-17.

71. "Le Corbusier y otras cuestiones", El Mundo (Argentina), 26 de septiembre de 1.966 , p. 46.

72. "De donde son los cantantes", Primera Plana (Buenos Aires), No. 251, 17 de octubre de 1967, pp. 56.57. De donde son los cantantes, pp. 91-102.

73. "Páginas en blanco", Margen (París), No. 5 (1967), Pp. 92-93.

74. "Cobra", Les Lettres Nouvelles, No. especial: Ecrivans de Cuba (Diciembre de 1967-enero de 1968), pp. 187-92.

75. 'L'Aventure (tex'uelle) d'un collectionneur des peaux (humaines)", Confrontations (París, Journal bimestral de la M.J.C. de la poterne des peuples), No. 1 (1968), p. 2. Escrito sobre an cuerpo, PP. 49.52.

76. "Los métodos de un crítico", [Sarduy entrevista a Emir Rodríguez Monegal], Imagen, Suplemento No. 30 [Sin fecha, 1968?]

77. "Cobra", Imagen, Suplemento No. 34, 1-15 de octubre de 1968.

78. "Gestos", Narraiva cubana de la revolución. Prólogo, selección y notas de J. M. Caballero Bonald. Madrid: Alianza Editorial, 1968, pp. 225-32. Gestos, pp. 19-30.

79. "La plage", L'Action Théâtrale, No. 1 (1969), pp. 15.17 [Frag. mento de pieza teatral.]

80. "Eat Flowers", Revist de da Universidad de México, 23 [No. 9] (1969), pp. 5.12. [Fragmento de Cobra.] 
81. "Poemas para el cielo", El Cielo (Buenos Aires), Año 1 [No. 3] (1969), pp. 2.7.

82. "Ruines: scènes en deux", L'Action Théâtrale, Nos. 5.6 (1970), pp. 22.23. [Fragmento de Ruines, pieza inédita representada en París.]

83. "A Dios dedico este mambo", El Urogallo, Nos. $5-6$ (1970), pp. 37.43. [Fragmento de Cobra.]

84. Interview avec Juan Goytisolo", L'Ari Vivant, No. 17 (1971), pp. 20-21.

85. "Para los pájaros", Papeles (Caracas), No. 14 (1971), pp. 8-21. [Fragmento de Cobra.]

86. "Gran mandala de las divinidades irritadas y detentoras del saber", Plural. Revishia mensual de Excelsior (México), No. 3 (1971), pp. 14-16. Prólogos y ensayos breves en catálogos de exposiciones de arte: [Fragmento de Cobra.]

87. "Matilla", Matill $l_{\mathfrak{a}}$ expone. Panfleto de la exposición del pintor cubano Julio Matilla que tuvo lugar en el Museo Ignacio Agra. monte, Camagüey, Cuba, del 21 al 28 de abril de 1957. [Una página sin numerar.]

88. Loló. 1953-1957. Panfleto de la exposición de la pintora cubana Dolores Soldevilla que tuvo lugar en la Sala de Exposiciones del Centro Profesional del Este, Caracas, Venezuela, el 17 de mayo de 1957. [Una página sin numerar.]

89. Catálogo invitación. 20 obras para una colección. Exposición de pintura y escultura de artistas de varios países celebrada en la Ga. lería Habana, La Habana, Cuba, diciembre de 1957. [Una página $\sin$ numerar.]

90. "Las topologias eróticas de "Zilia Sánchez", Estruchuras en secuencia. Panfleto de la exposición de la pintora cubana celebrada del 8 al 23 de septiembre de 1970 en el Museo de la Universidad de Puerto Rico. [Tres páginas sin numerat.]

91. "matière/machines/phantasme/ciel", Alejandro. Catálogo de la ex. posición del pintor cubano José Ramón Díaz Alejandro celebrada en la Galería Jacques Desbrière, París, del 16 de febrero al 10 de marzo de 1971. [Dos páginas sin numerar.] 


\section{BIBLIOGRAFIA SOBRE SEVERO SARDUY}

\section{Entrevistas:}

92. Rodríguez Monegal, Emir. "Las estructuras de la narración. Diálogo", Mundo Nuevo, No. 2 (1966), pp. 15-26. Recogida en el libro de Rodríguez Monegal, El arte de narrar. Caracas: Monte Avila, 1968, pp. 269-92.

93. Papastamatiu, Basilia. "Entrevista con Severo Sarduy. Una nueva interpretación del barroco", Imagen (Caracas), Suplemento Nos. 14.15 (1967).

94. Zalaffi, Nicoletta, y François Truchaud. "Qui êtes-vous, Severo Sarduy", MédicinelCinéma, No. 5 (1969), pp. 40.45.

95. Hurtado, Efraín. "Entrevista con Severo Sarduy", Actual. Revisia de la Universidad de los Andes, Año 2 [No. 5] (1969), pp. 123-28.

96. Fossey, Jean Michel. "Severo Sarduy par lui même", Cormorán (Santiago de Chile), Año 1 [No. 6] (1970), pp. 4:5.

97. 'Severo Sarduy; una nebulosa información", Confirmado (Buenos Aires, 29 de abril de 1970, pp. 44-45. [Yulan M. Washburn reprodujo parcialmente esta entrevista en Hispania, 53 (1970), p. 1016.]

98. Fell, Claude: "Entretien avec Severo Sarduy”, Le Monde, 26 de septiembre de 1970, p. 7.

99. Rodriguez Monegal, Emir. "Conversación con Severo Sarduy", Revista de Occidente, No. 93 (1970), pp. 315-43.

100 Fossey, Jean'-Michel. "Entrevista con Severo Sarduy", Imagen (Caracas), Nos. 94-95 (1971), pp. 28-29.

Estudios, reseñas y menciones:

101. Portuondo, José Antonio. Bosquejo bistórico de las letras cubanas. La Habana: Editorial del Ministerio de Educación, 1962, p. 73.

102. Henríquez Ureña, Max. Panorama bistórico de la literatura cubana (1492-1952). Nueva York: Las Américas, 1963, Vol. II, p. 447.

103. Estange, Luc. "Gestes, roman cubain sur thytme de cha-cha-cha", Le Figaro Littéraire, 22 de junio de 1963, p. 5.

104. Ricaumonde, Jacques de. "Gestes de Severo Sarduy", Combat, 11 de julio de 1963, p. 7. 
105. Piafier, Jacqueline. "Gestes, de Severo Sarduy", Le Monde, 13 de julio de 1963, p. 11.

106. Murena, Héctor A. "Debut de Severo Sarduy en la novela", Siete dias. Revista Dominical de La Prensa (Lima), No. 355, 22 de noviembre de 1964, p. 27.

107. Portuondo, José Antonio. Críticad de la época y otros ensayos. Santa Clara: Editorial del Consejo Nacional de Universidades, Universidad de Las Villas, 1965, pp. 200-01.

108. Barthes, Roland. Critique et vérité. París: Editions du Seuil, 1966, p. 75.

109. Schulman, Ivan A. "La situatión y Gesłos: dos técnicas y dos visiones de la realidad cubana", Duquesne Hispanic Review, 5 (1966), pp. 121.23.

110. Fornet, Ambrosio. "New World en español", Casa de las Américas (La Habana), Año 7 [No. 40] (1967), pp. 106-15.

111. Barthes, Roland. "Sarduy: la face baroque", La Quinzaine Littéraire, No. 28 (1967), p. 13. En español: "Sarduy: la faz de barroca", Mundo Nuevo, No. 14 (1967), pp. 70.71.

112. Morelle, Paul. "Une littérature 'gestuelle': Ecrit en dansant", Le Monde, 24 de mayo de 1967, Supplément, p. 2.

113. Couffon, Claude. "Severo Sarduy et la réalité cubaine", Les Letrros Françaises, 22 de mayo de 1967, pp. 9-10. En español: "Sarduy y la realidad cubana", Mundo Nuevo, No. 22 (1968), pp. 87-88.

114. Velan, Yves. "Moments littéraires: Ecrit en dansant", La Gazefte de Lausanne, 27.28 de mayo de 1967.

115. Henric, Jacques. "Cuba, l'île lue", France Nouvelle, 16 de agosto de 1967, p. 13.

116. Penel, Alain. "Cuba taconté par une cigale: Ecrit en dansanu", La Tribune de Genève, 26.27 de agosto de 1967, p. 5.

117. R[udni], S[ilvia]. "Severo Sarduy o el idioma que baila", Primera Plana (Buenos Aires), No. 256, 21 de noviembre de 1967, p. 70.

118. Sáinz, Gustavo. "De donde son los cantantes", El Día (México), 27 de enero de 1968, p. 9.

119. Sucre, Guillermo. "Severo Sarduy: los plenos poderes de la retórica", Imagen (Caracas), Suplemento No. 20 (1968), p. 24.

120. Castro Arenas, Mario. "Novelistas cubanos artistas del idioma", Siele dias. Revista Dominical de La Prensa (Lima), No. 511, 7 de abril de 1968, pp. 28-29.

121. Peña, Margarita. "De donde son los canziantes", Díallogos (México), 4 [No. 21] (1968), pp. 36.37. 
122. Cozarinski, Edgardo. "Un espacio verbal llamado Cuba", Sur (Buenos Aires), No. 312 (1968), pp. 69.70.

123. Campos, Julieta. "Mamá yo quiero saber en dónde se experimenta", Siempre! (México), 6 de junio de 1968.

124. Anónimo. "Cuban Rum". The Tims Litierary Supplement (Lon. dres), No. 3,481, 14 de noviembre de 1968, p. 1.270 .

125. Anónimo. "Libros, el sueño de Severo Sarduy", Siempre! (México), 3 de abril de 1968, pp. 14-15.

126. Pagés Larraya, Antonio. "Una novela de Severo Sarduy", Comen. tario (Buenos Aires), Año 16 [No. 65] (1969), pp. 55.57.

127. S[hoo], E[rnesto]. "Severo Sarduy: cuerpos y libros", Primera Plana (Buenos Aires). No. 336, 3 de junio de 1969, pp. 58.59.

128. Aguilar Mora, Jorge. Cobra, barroco y otras palabras", Siempre! (México), 25 de junio de 1969, pp. 7-8.

129. Martínez, Tomás Eloy. "El lenguaje, ese libidinoso", Primera Plana (Buenos Aires), No. 340, 1 de julio de 1969, p. 58.

130. Briante, Miguel. "Falsas notas/homenaje a..." Confirmad'o (Buenos Aires), 24 de julio de 1969, p. 10.

131. Murena, Héctor A. "Un novelista joven, sutil y vigoroso", La Voz del Interior (Argentina), 1 de noviembre de 1969 p. ?. En francés: "Un romancier jeune, plein de sensibilité et de vigueur", Les Letres Nouvelles, 13 année [Nouvelle série] (1970), pp. 139-42.

132. Ortega, Julio. "Nota sobre Sarduy", La contemplación y la fiesta. Caracas: Monte Avila, 1969, pp. 205.11.

133. Miranda, Julio E. "Escrito en cubano", Imagen (Caracas) N'o. 74 (1970), p. 10.

134. Brandeau, Michel. 'La génération illisible' ou l'avant garde littéraire", Actuel!?, no. 3 [Nouvelle série mensuelle] (1970), pp. 46-47.

135. Sollers, Philippe, "La boca obra", Tel Quel, No. 42 (1970), pp. 46.47 .

135. Sollers, Philippe, "La boca obra", Tel. Quel, No. 42 (1970), pp. 35-36.

136. Miranda, Julio E. "El nuevo pensamiento cubano", El Urogallo, 2 [No. 8] (1971), pp. 84-93.

137. Feito, Francisco E. "Severo Sarduy en la actual narrativa hispanoamericana", El Urogallo, 2 [No. 8] (1971), pp. 94-97.

138. Conte, Rafael. "Severo Sarduy, entre el juego y la transgresión", Informaciones (Madrid), 15 de abril de 1971, pp. 1-2. 
139. Rosa, Nicolás. "La crítica como metáfora", Vanguardia Dominical (Bucaramanga, Colombia), 23 de mayo de 1971, p. 10.

140. Culleré, Carlos A. "Severo Sarduy: un mundo inédito", Papel Literario de El Nacional (Caracas), 29 de agosto de 1971.

Addenda:

Sobre la representación de la obra teatral La Playa pueden consultarse las reseñas aparecidas durante la primavera de 1971 en los siguientes periódicos alemanes: Hassesche Allgemeine (Cassel), Der Tagesspiegel (Berlín), Frankfurter Allgemeine (Frankfort), Die Welt (Hamburgo) y Süddeutscbe Zeitung (Munich).

De Sarduy hay los siguientes trabajos en prensa: Cobra (novela, Sud. americana), La plage (pieza teatral, Cahier Renault-Barrault), fragmen. tos de Cobra en Libre (París), y un trabajo de crítica en Plural (México).

Sobre Severo Sarduy hay los siguientes trabajos en prensa: Ana María Barrenechea, "Severo Sarduy o la aventura textual", en Proceedings of Colloquium on the Latin American Narative (Chapel Hill, North Carolina, 1971), que será publicado por University of Nortb Carolina Studies in Romance Languages and Literatures; Roberto González Echevarría, "Son de La Habana: la ruta de Severo Sarduy", Revisaa Ibenoamericana y "A Conversation with Severo Sarduy", Diacritics, que aparecerá en castellano en Papeles (Caracas). 
